this method should not be considered as a contraindication (4).

Conclusions PSB can be safely applied without increasing morbidity and avoiding possible complications that may arise from the results of other anesthesia approaches.

\section{Postoperative pain management}

\section{PAIN MANAGEMENT AFTER COMPLEX SPINE SURGERY: A SYSTEMATIC REVIEW AND PROCEDURE-SPECIFIC POSTOPERATIVE PAIN MANAGEMENT (PROSPECT) RECOMMENDATIONS}

${ }^{1} \mathrm{P}$ Waelkens ${ }^{*},{ }^{2} \mathrm{E}$ Alsabbagh, ${ }^{3} \mathrm{~A}$ Sauter, ${ }^{4} \mathrm{G}$ Joshi, ${ }^{2} \mathrm{H}$ Beloeil. ${ }^{1}$ University Hospitals Leuven, Leuven, Belgium; ${ }^{2} \mathrm{CHU}$ Rennes, Rennes, France; ${ }^{3}$ University of Oslo, Oslo, Norway; ${ }^{4}$ University of Texas Southwestern Medical Center, Dallas, USA

\subsection{6/rapm-2021-ESRA.211}

Background and Aims Complex spinal procedures are associated with intense pain in the postoperative period. Adequate perioperative pain management has been shown to correlate with improved outcomes including early ambulation and discharge. We aimed to evaluate the available literature and develop recommendations for optimal pain management after complex spine surgery.

Methods A systematic review using the PROSPECT methodology was undertaken. RCTs and systematic reviews published in the English language from January 2008 to April 2020 assessing postoperative pain after complex spine surgery using analgesic, anaesthetic or surgical interventions were identified from MEDLINE, EMBASE and Cochrane Databases.

Results Out of 111 eligible studies identified, 31 RCTs and 4 systematic reviews met the inclusion criteria. Preoperative and intraoperative interventions that improved postoperative pain were paracetamol, COX-2 specific-inhibitors or NSAIDs, intravenous ketamine infusion and regional analgesia techniques

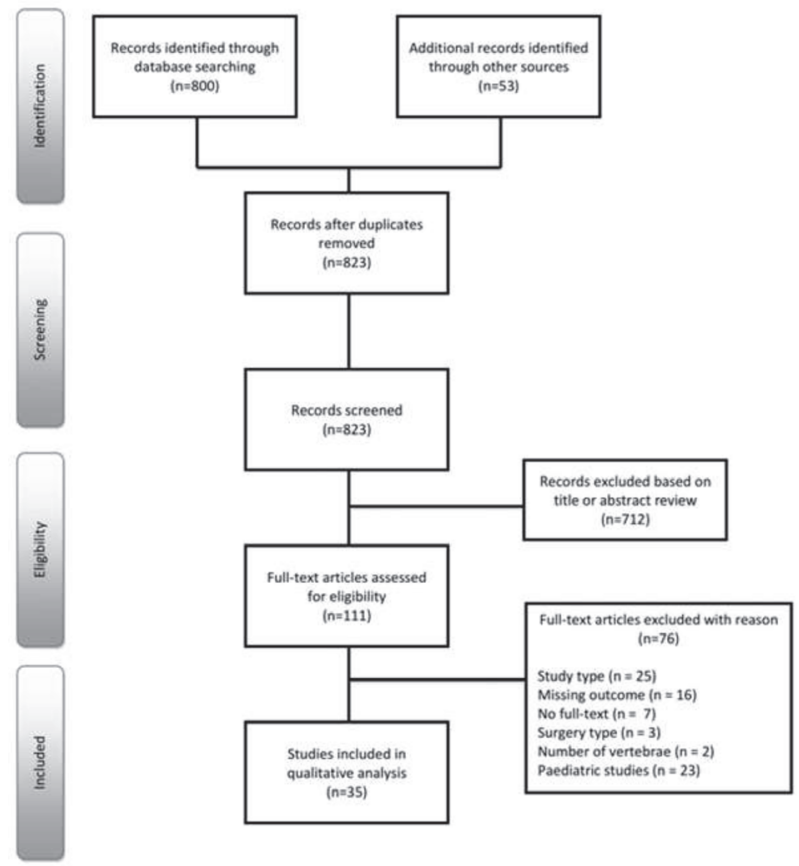

Abstract 211 Figure 1 including epidural analgesia using local anaesthetics with or without opioids. Limited evidence was found for local wound infiltration, intrathecal and epidural opioids, erector spinae plane block, thoracolumbar interfacial plane block, intravenous lidocaine, dexmedetomidine and gabapentin.

Conclusions The analgesic regimen should include preoperative or intraoperative paracetamol and COX-2 specific inhibitors or NSAIDs, continued postoperatively with opioids used as rescue analgesics. Other recommendations are intraoperative ketamine and epidural analgesia using local anaesthetics with or without opioids. Although there is procedure-specific evidence in favour of intraoperative methadone, it is not recommended as it was compared with shorter-acting opioids and due to its limited safety profile. Further qualitative RCTs are required to confirm the efficacy and safety of these recommended analgesics on postoperative pain relief.

\section{IMPACT OF INTRATHECAL DIAMORPHINE IN ENHANCED RECOVERY FOR COLORECTAL SURGERY; OPEN AND LAPAROSCOPIC}

J Luyt*, D Wagstaff, WL Yap. Countess of Chester Hospital, Chester, UK

\subsection{6/rapm-2021-ESRA.212}

Background and Aims There is currently a paucity of evidence in the utility of intrathecal diamorphine for resectional bowel surgery. We audited our departmental practice for perioperative analgesia in the provisions of anaesthesia services for bowel resections.

We looked at the use of a neuraxial block with intrathecal diamorphine, to determine if it has a positive impact on enhanced recovery. Specifically; post-operative opiate requirements, eating and drinking, and mobilising within 24 hours after surgery.

Methods A cohort of randomised patients were selected as per Perioperative Quality Improvement Project (PQIP) data collection protocol from June 2018 to June 2019, 48 patients were eligible. Data was collected from anaesthetic charts, electronic prescribing, and PQIP database. Criteria for data collection included intra- and post-operative analgesia; drinking, eating and mobilising within the first 24 hours of surgery.

Results $77 \%(\mathrm{n}=37)$ of patients received intrathecal diamorphine perioperatively.

Patient controlled analgesia (PCA) morphine consumption is higher on average, $44 \mathrm{mg}$ : $22 \mathrm{mg}$, in non-spinal patients.

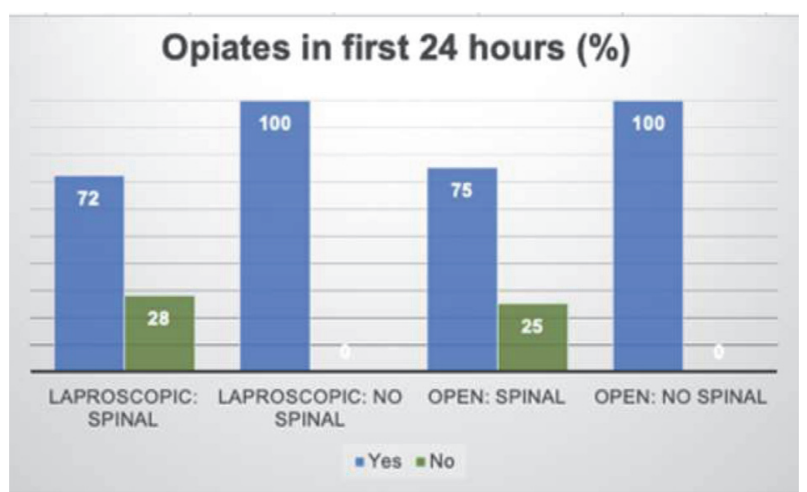

Abstract 212 Figure 1 